1950 s and has been widely adopted in education systems. Although titled 'Forest schools' it is the philosophy which is more important than the forest environment per se. It is based on regular exposure to a natural environment which offers children the opportunity to take appropriate risks and gain resilience, confidence and independence as creative learners. Improved outcomes include:

- Increased self-esteem and self-confidence

- Improved social skills

- The development of language and communication skills

- Improved physical motor skills

- Improved motivation and concentration

- Increased knowledge and understanding of the environment

Conclusions The benefits of the natural environment is little surprise considering the human race coexisted with nature for 99.99\% of its evolution. The Biophilia Hypothesis recognises the biological attraction of nature to human beings and promotes the benefits of intimate contact with nature. Preliminary results suggest that contact with nature should be encouraged in health and education environments.

\section{G174(P) ELECTRIC SCOOTERS, THEIR SAFETY AND THE LAW AROUND THEIR USE IN THE UK}

${ }^{1} \mathrm{G}$ Guiton, ${ }^{2} \mathrm{~F}$ Finlay. ${ }^{1} \mathrm{~F} 1$ Doctor, Dorset County Hospital, Dorchester, UK; ${ }^{2}$ Community Child Health, Virgin Care, St Martins Hospital, Bath, UK

\subsection{6/archdischild-2020-rcpch. 145}

Aims To review the popularity of electric (E) scooters, their safety and the law around their use in the UK.

Methods A review of the literature.

Results E-scooters are rising in popularity in both children and adults and have the potential to provide an attractive solution to common urban mobility problems - they are fun to use, cheap to run, improve mobility around cities, reduce congestion levels and do not use traditional fuels which have severe environmental implications. They are fitted with rechargeable batteries and have speeds ranging from $9 \mathrm{mph}$ to $15 \mathrm{mph}$.

Sharing schemes are popular and available in over 100 cities in Europe and US, users collecting them from various points around a city, renting them using an app and docking them when no longer required. In 2018, 84 million trips were taken on shared micromobility in the US - more than double the number of trips in 2017.

However in the UK the picture is very different. E-scooters cannot be legally ridden on the road or in cycle lanes and the Highway Act 1835 (originally written for horses and carts!) also prohibits their use on a pavement. They may only be legally ridden on private land with the permission of the landowner. This is due to their classification as 'powered transporters' or 'personal light electric vehicles' by Department for Transport (DfT) making them subject to motor vehicle laws, including the need for insurance, license, tax, MOT, visible rear red lights and number plates.

The design of E-scooters makes them portable, light and efficient but also makes them unsafe. They have two small tyres their user being inches from the road surface, resulting in a significant risk of traumatic injury, particularly when going over drain covers or potholes. Few wear helmets when riding them and there have been numerous injuries and deaths reported in various countries.
Conclusion With the shift to become more environmentally aware a 'future of mobility review' is considering how new types of vehicles, E-scooters, will change transport. It will also explore whether current laws should be changed to facilitate innovation while prioritising safety. Children need to be aware of the current laws with regard to their use and should be encouraged to wear helmets.

\section{Children's cancer and leukaemia group (CCLG) and paediatric special interest group: british society of haematology}

\section{Plenary}

\section{P05 WHAT ARE THE SIGNS AND SYMPTOMS OF BONE TUMOURS IN CHILDHOOD? ARE THE GREAT BRITISH PUBLIC AWARE OF THEM?}

${ }^{1} \mathrm{D}$ Shanmugavadivel, ${ }^{1} \mathrm{JF}$ Liu, ${ }^{2} \mathrm{~A}$ Stewart, ${ }^{2} \mathrm{~A}$ Gamble, ${ }^{1} \mathrm{D}$ Walker. ${ }^{1}$ Children's Brain Tumour Research Centre, University of Notitngham, Nottingham, UK; ${ }^{2}$ Children's Cancer and Leukaemia Group, CCLG, Leicester, UK

\subsection{6/archdischild-2020-rcpch.146}

Aims Bone tumours in children/young people are often associated with significant diagnostic delay, increasing the risk of amputation. The total diagnostic interval is a sum total of the patient and system interval. The HeadSmart campaign showed a national awareness campaign based on high quality evidenced based guidance halved time to diagnosis. We conducted a systematic review and meta-analysis to identify the core signs/symptoms and a public survey to understand public awareness of these symptoms.

Methods Medline, Pubmed and Embase databases were searched from January 2005 - August 2015. Key words included 'bone tumour(s)', 'bone neoplasm(s)', 'diagnosis', 'signs(s)', 'symptom(s)', 'presentation(s)'. All papers discussing bone tumour presentation were included. Pooled proportions (\%) of children with each sign/symptom at diagnosis were estimated. A face-to-face opinion survey ( $\mathrm{n}=1000 ; 475$ males) was conducted by Ipsos MORI. Questions included perception of cancer risk, confidence in recognising signs/symptoms and urgency of seeking medical advice.

Results The search strategy identified 15477 papers. 713 papers were reviewed in full; 11 met the inclusion criteria, describing the symptoms/signs at diagnosis in 1246 children. 29 symptoms/signs were recorded but only those that occurred in $2 \%$ or more of patients are reported. These were pain (76\%), swelling (21\%), fever (4\%), history of trauma (3\%), functional limitation (3\%), palpable mass $(3 \%)$, pain and swelling $(2 \%)$, volume increase $(2 \%)$, limp $(2 \%)$ and pathological fracture $(2 \%)$. Public awareness of the top 4 presenting symptoms of bone tumours were low. $23 \%$ of the public identified recurrent or persistent bone pain as a symptom of cancer; $27 \%$ identified swelling of bone or joint; $21 \%$ identified fever and $14 \%$ thought a slow recovery after injury or trauma could be a sign of cancer.

Conclusions This is the first systematic review on paediatric bone tumours to our knowledge. It provides strong evidence of the common presentations and will form the basis of a new RCPCH endorsed clinical guideline on the assessment/ investigation of suspected bone tumours. The results of the 
public awareness survey highlight low awareness which need to be tackled in order to drive earlier diagnosis. This will form key messaging in our new national awareness campaign called ChildCancerSmart.

\section{G176 PUBLIC AWARENESS OF CHILDHOOD, TEENAGERS AND YOUNG ADULT CANCER SIGNS AND SYMPTOMS IN GREAT BRITAIN}

${ }^{1}$ JF Liu, 'D Shanmugavadivel, ${ }^{2} \mathrm{~A}$ Gamble, ${ }^{2} \mathrm{~A}$ Stewart, ${ }^{1} \mathrm{DA}$ Walker. ${ }^{1}$ Children's Brain Tumour Research Centre, University of Nottingham, Nottingham, UK; ' ${ }^{2}$ Children's Cancer and Leukaemia Group, Leicester, UK

\subsection{6/archdischild-2020-rcpch. 147}

Aims To assess public awareness of the risks and symptoms of cancer in children and young adults under 18 in Great Britain and compare them with the published data where $60-94 \%$ of adults are aware of their cancer risks and symptoms.

Methods A face-to-face, computer-assisted opinion survey was conducted by Ipsos MORI. The population-based sample included 475 males and 525 females over the age of 18 and $26 \%$ have children aged 6-15 in the household. Questions covered perception about cumulative cancer risk ( 1 in 450 by the age 15 and 1 in 180 by 25), confidence in recognising signs and symptoms, recognition and perceived urgency of 42 classical signs and symptoms.

Results Over half (56\%) of the respondents felt the quoted cumulative age-related cancer risks were higher than they expected. Only $32 \%$ felt confident to recognise the signs and symptoms of cancer in the age range. On average, respondents identified 14.9 out of the 42 of the classical signs and symptoms. Top ranked symptoms or signs were: 'lump, swelling in pelvis, testicle or breast' (46\%), 'blood in urine or stool' $44 \%$, 'changes to moles' $43 \%$, 'lump or swelling in the chest wall or armpits' $41 \%$ and 'weight loss' $40 \%$. The least recognised symptoms were early or late puberty (10\%), developmental delay in young children under $2(11 \%)$ and slow growth (13\%), where $8 \%, 2 \%$ and $6 \%$, respectively, felt that there was no need to discuss them with a doctor. Symptoms where more than $50 \%$ of respondents judged them as requiring medical assessment within 48 hours were: seizures/fits, blood in urine or stool, persistent vomiting, persistent fever or night sweats. Abnormal facial movements and persistent abdominal pain were added for assessment within 2 weeks. All but one of the symptoms were selected for assessment by 3 months.

Conclusions We conclude that public awareness of the risks and signs and symptoms of childhood cancer are substantially lower than awareness for adult cancer population in Great Britain. A similar survey will be conducted amongst healthcare professionals to support the development of campaign materials for a public and professional awareness programme.

\section{G177 CISPLATIN-INDUCED OTOTOXICITY IN PAEDIATRIC AND ADOLESCENT OSTEOSARCOMA PATIENTS REMAINS A CHALLENGE}

C Soto, EA Corley, M Michelagnoli. Children and Young People's Cancer Service, UCL Hospitals, London, UK

10.1136/archdischild-2020-rcpch. 148
Background/Objectives Cisplatin-induced ototoxicity is a wellrecognised complication of cancer treatment, resulting in nonreversible, often progressive hearing loss.

Risk factors for cisplatin-induced hearing loss include higher cumulative cisplatin dose, younger patient age, head/neck radiotherapy and concomitant use of aminoglycosides. Osteosarcoma typically affects long-bones in later childhood and adolescence; radiotherapy has a limited role and local protocols prohibit routine use of aminoglycosides. We examined whether cisplatin-induced ototoxicity remained a significant side-effect in this lower risk patient group in a retrospective cohort of patients treated as per the EURAMOS-1 protocol (cumulative cisplatin dose $480 \mathrm{mg} / \mathrm{m}^{2}$ ).

Methods Paediatric and adolescent osteosarcoma patients at a single UK tertiary centre over a five-year period (2013 2017) were included. Clinical information and results of puretone audiograms were extracted from patient records. Common Terminology Criteria for Adverse Events version 5 definitions were used to describe ototoxicity; extended high frequency hearing loss (EHFHL) was defined as range $>$ $8 \mathrm{KHz}$.

Results We identified 72 eligible patients with mean age of 12.9 years (median 16 years, range $2-18$ years) and equal gender distribution. 3 patients progressed/relapsed prior to cycle 4 and a further 3 progressed/relapsed prior to their end of treatment assessment. Baseline audiometry was recorded in 59 patients $(82 \%)$.

Of 53 patients who had audiometry before their 4th cycle of cisplatin; EHFHL was seen in 13 (25\%), 6 (11\%) had Grade 1 and 7 (13\%) had Grade 2 hearing loss. 37 patients underwent an end of treatment (EOT) audiogram; 13 (35\%) had some degree of hearing loss and $5(13 \%)$ had grade $\geq 3$. Of the patients with demonstrated hearing loss after three cycles of cisplatin, who had an EOT assessment, 6 demonstrated worsening audiometry.

Conclusion The finding that over half the patients in this lower risk cohort experienced some degree of hearing loss at end of treatment illustrates that ototoxicity remains a significant complication of cisplatin treatment and highlights the importance of rigorous audiometry throughout and following EOT. Since cisplatin has a central role in osteosarcoma prompt recognition of ototoxicity and early institution of support measures is essential and, ultimately, management options to minimise risk of ototoxicity are urgently needed.

\section{G178 LOW-LEVEL LASER THERAPY FOR CHILDREN AND YOUNG PEOPLE UNDERGOING TREATMENT FOR CANCER}

${ }^{1,2} \mathrm{M}$ Redman, ${ }^{3} \mathrm{~K}$ Harris, ${ }^{4} \mathrm{~B}$ Phillips. 'Oncology and Metabolism, University of Sheffield, Sheffield, UK; ${ }^{2}$ Paediatrics, Sheffield Children's NHS Foundation Trust, Sheffield, UK; ${ }^{3}$ Medical Education, University of Liverpool, Liverpool, UK; ${ }^{4}$ Centre for Reviews and Dissemination, University of York, York, UK

\subsection{6/archdischild-2020-rcpch. 149}

Aim To assess the efficacy and tolerance/safety of oral lowlevel laser therapy to prevent or treat oral mucositis in children and young people undergoing chemotherapy.

Introduction Inflammation and ulceration of the oral cavity known as oral mucositis - can affect up to $80 \%$ of children and young people (CYP) undergoing chemotherapy. It can lead to severe oral pain as well as difficulty talking, eating and drinking; it may also lead to treatment cessation. 